\title{
INTRACARDIAC ELECTROGRAPHY IN THE INVESTIGATION OF CONGENITAL HEART DISEASE IN INFANCY AND THE NEONATAL PERIOD
}

\author{
BY \\ HAMISH WATSON \\ From the Department of Medicine, University of St. Andrews, Queen's College, Dundee \\ Received August 20, 1961
}

Though cardiac surgery has advanced rapidly and provided a constant stimulus to the development of precise diagnostic methods, the highest mortality from congenital heart disease is still in the first year of life and there is, as yet, no suitably sized pump oxygenator available for very small children. There seems little doubt, however, that before long a satisfactory machine will be developed and we should, therefore, in anticipation, be modifying and adapting our diagnostic methods to suit infants and very young children who present special problems to the cardiologist. Many of them are ill or in heart failure and intracardiac manipulations have to be very gentle in such casesthe heart being only about the size of a walnut and often very irritable. Cardiac catheterization may have to be abandoned at any stage, so speed is essential and any method of reducing the length of the investigation is of value. This paper illustrates a few of the ways in which the routine use of intracardiac electrography can make cardiac catheterization in infants and the newborn quicker, safer and more informative.

\section{METHOD}

Intracardiac electrograms are easily obtained using standard production United States Catheter and Instrument Corporation catheters. Similar to Cournand single lumen catheters with the addition of an electrode ring flush with the surface one or two millimetres from the distal tip, they are obtainable in all sizes from $5 \mathrm{~F}$ to $14 \mathrm{~F}$ and 50,100 or $125 \mathrm{~cm}$. long. A wire conductor runs down spirally in the wall from the electrode to a terminal contact at the proximal end of the catheter, and some simple device is required to connect this to suitable recording apparatus. Many small technical difficulties may be encountered before good reproducible records are obtained, but most of them are easily overcome-careful suppression of A.C. interference and the maintenance of a completely dry field are probably the most important.

The intracardiac electrogram is useful both during cardiac catheterization, when it can be monitored on an oscilloscope either alone or with simultaneously recorded pulses, and also later when the photographic records made during the investigation are reported (Emslie-Smith, 1955). Complexes may be recorded either with the catheter tip in a relatively static position or as it moves about inside the heart and great vessels. Many of the illustrations in this paper are tracings made as a catheter is withdrawn slowly from one site to another and as catheterization is in all cases from the right groin, the withdrawals are in that direction.

Intracardiac potentials are often of high voltage and at normal standardization very large QRS complexes may be recorded in the ventricles. Wide photographic film should be used to ensure that they do not cross or become mixed up with other simultaneously recorded signals making interpretation difficult. For routine work the intracardiac lead is best standardized at 2 millivolts per $\mathrm{cm}$. and sensitivity can be increased to record low voltage complexes when necessary. Fast paper speeds may be required for detailed study of individual deflections and a good light and some 
magnification help with the analysis of high speed deflections which may require a little emphasis to make them visible in illustrations.

Many nomenclatures have been used to designate the various deflections recorded by the intracardiac electrode in different parts of the heart, and confusion has arisen because some authors have used the terms auricular and ventricular to designate atrial and ventricular activity wherever it happens to be recorded, others to denote the total electrical activity recorded in either chamber. A ventriculogram may thus be either any QRS complex inside the heart and great vessels or the $\mathrm{P}, \mathrm{QRS}$, and $\mathrm{T}$ waves recorded in the ventricle itself. In this paper the terms atriogram and ventriculogram are used to refer to the whole sequence of electrical events recorded during the cardiac cycle in the atrium and ventricle respectively.

\section{Atriograms}

Because the heart is small, it is not always easy to tell on screening exactly where the catheter is. The tip often crosses the mid-line fairly high in the right atrium and when it will not pass easily

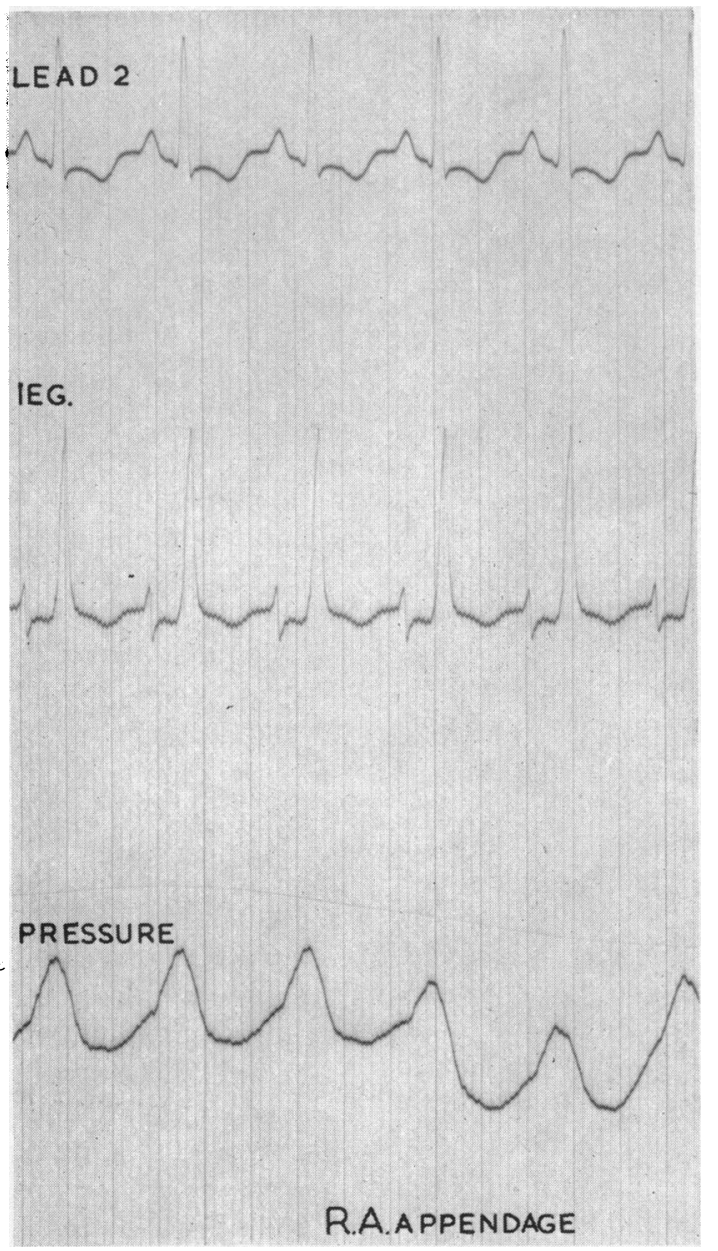

FIG. 1.-A record made in the right atrial appendage showing the characteristic intracardiac electrogram (IEG) with tall $R$ as the sole deflection and an inverted $T$ wave. into a pulmonary vein and it is not certain whether the left atrium has been entered or not, much time can be saved if, instead of probing, stopping to take pressure records or blood samples, the intracardiac electrogram is inspected on the oscilloscope. Often the characteristic complexes illustrated in Fig. 1 will be seen, indicating that the electrode is in the right atrial appendage which is sometimes very mobile and can move far from its normal anatomical position with a catheter in its apex. While attempting to pass a catheter through an atrial septal defect it is thus possible to tell each time whether the tip has been advanced into the left atrium or merely into the right auricle.

The intracardiac lead can also confirm the presence of defects in the interatrial septum and at the same time help to determine their site. The movement of the base line on the recording of the intracardiac electrogram in Fig. 2 is a very constant feature of tracings made during cardiac catheterization from the groin as the catheter tip is withdrawn slowly from left to right atrium. It is seldom seen when catheterization is from the arm and is of course an artefact caused by the sudden movement of the catheter tip as it comes across the edge of the defect, and similar to that which would be seen in a strip of standard lead II if the patient moved his right arm during the recording. Fig. 2 has been selected from a case where the left and right atriograms are very different, but when they are similar, and they often are, this flip can be helpful, especially if, as here, there is little change in the recorded pressure pulses.

When an electrode passes down through the atrium there is a gradual and constant change in 


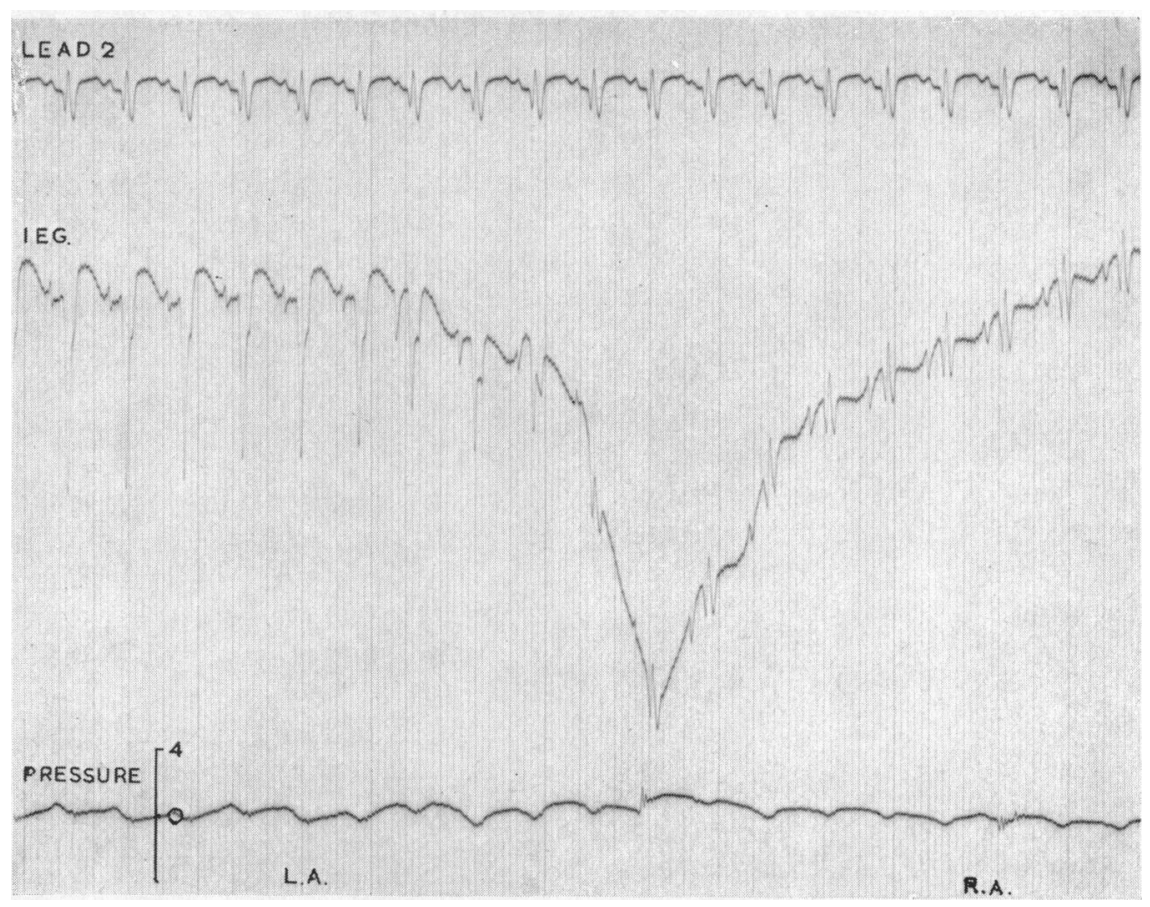

FIG. 2.-A withdrawal tracing from left to right atrium in a case of atrial septal defect showing that as the tip of the catheter comes across the edge of a defect there is sudden movement of the base line of the intracardiac electrogram. This catheter flip is a fairly constant feature when withdrawals are made from the groin and pin-points the site of the defect on the record.

the polarity of the $\mathrm{P}$ waves, which are negative high in the atrium, become diphasic in mid cavity and positive low in the chamber (Fig. 3). Using this knowledge in conjunction with the catheter movement artefact, we can get some idea of the site of the defect in the interatrial septum. In the record seen in Fig. 4, the tip of the catheter has been pressing initially against the lateral wall of the left atrium producing atrial injury or contact currents, with consequent damping of the pressure pulse tracing. As withdrawal begins contact is lost, the $\mathbf{P}_{T}$ elevation disappears and the left atrial pressure pulse reappears. When the catheter tip moves from left to right atrium the flip pin-points the site of the defect on the record and the $\mathrm{P}$ waves on either side of it can then be studied. In this case they are biphasic in the left atrium and biphasic in the right atrium-suggesting that the catheter tip has crossed from left to right in the mid septal area, through a secundum type defect.

In infants it is the rule rather than the exception for the catheter to cross into the left atrium, and it nearly always does so through a foramen ovale rather than through a true septal defect. This method of analysis gives valuable confirmatory evidence that the route has been via the foramen ovale and an almost constant pattern is produced on withdrawal tracings. In Fig. 5, for example, the catheter tip has initially been screened into mid left atrium, where pressures and oxygen saturations have been recorded, the position being confirmed by the biphasic nature of the $P$ waves in the atriogram. Withdrawal from left to right atrium begins and, as would be expected when the catheter has pushed its way through a foramen ovale fairly high in the septal wall, it has to come back up into the upper part of the left atrium again on its way out - the $P$ waves changing as it goes from the biphasic ones of mid cavity to the wholly negative ones in the upper part of the chamber. The catheter then slips back through the foramen into the right atrium and the catheter flip produces the usual baseline movement. Because of this wandering of the iso-electric line it is impossible to 
LEAD 2

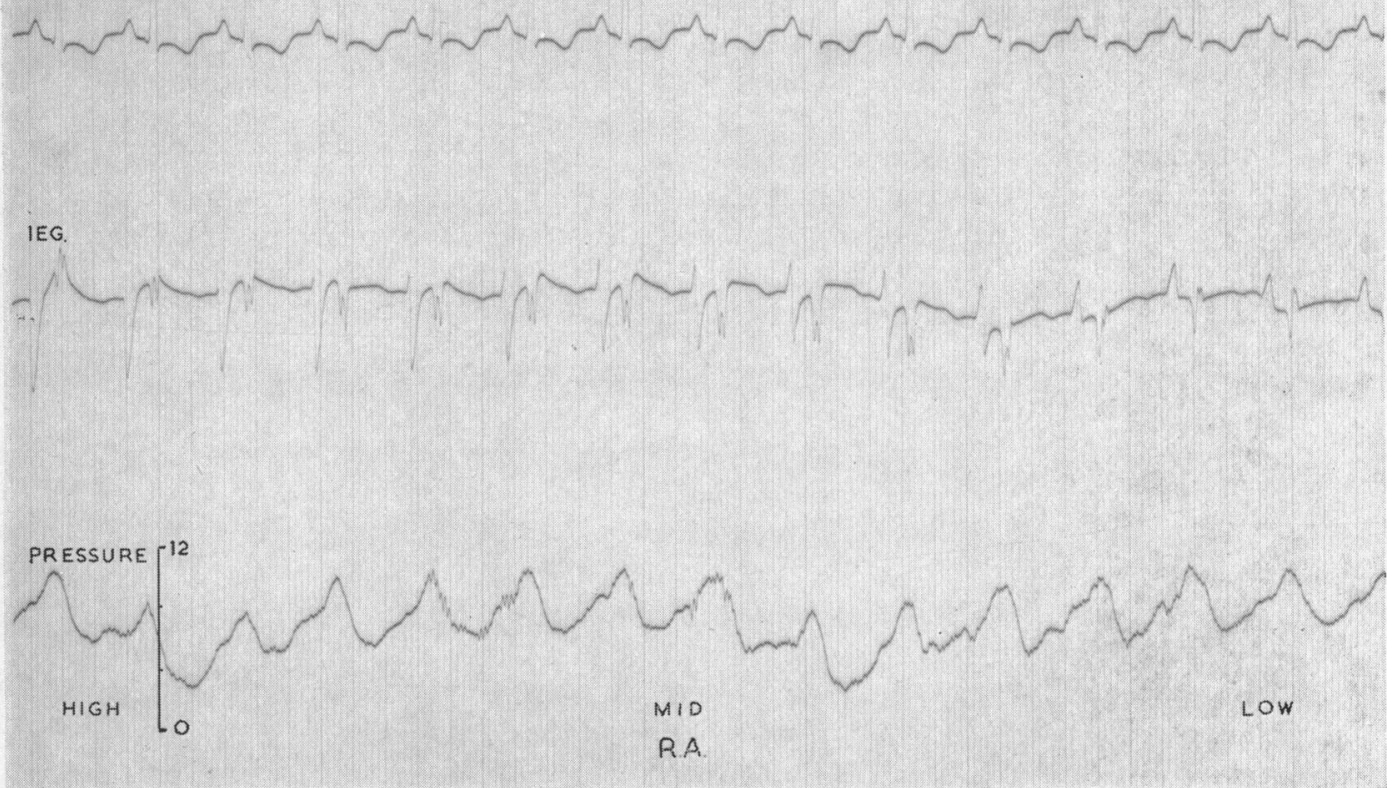

FIG. 3. - Showing that as an electrode passes down through the right atrium there is a gradual change in the polarity of the $\mathbf{P}$ waves which are negative high in the atrium, biphasic in mid cavity and positive low in the chamber.

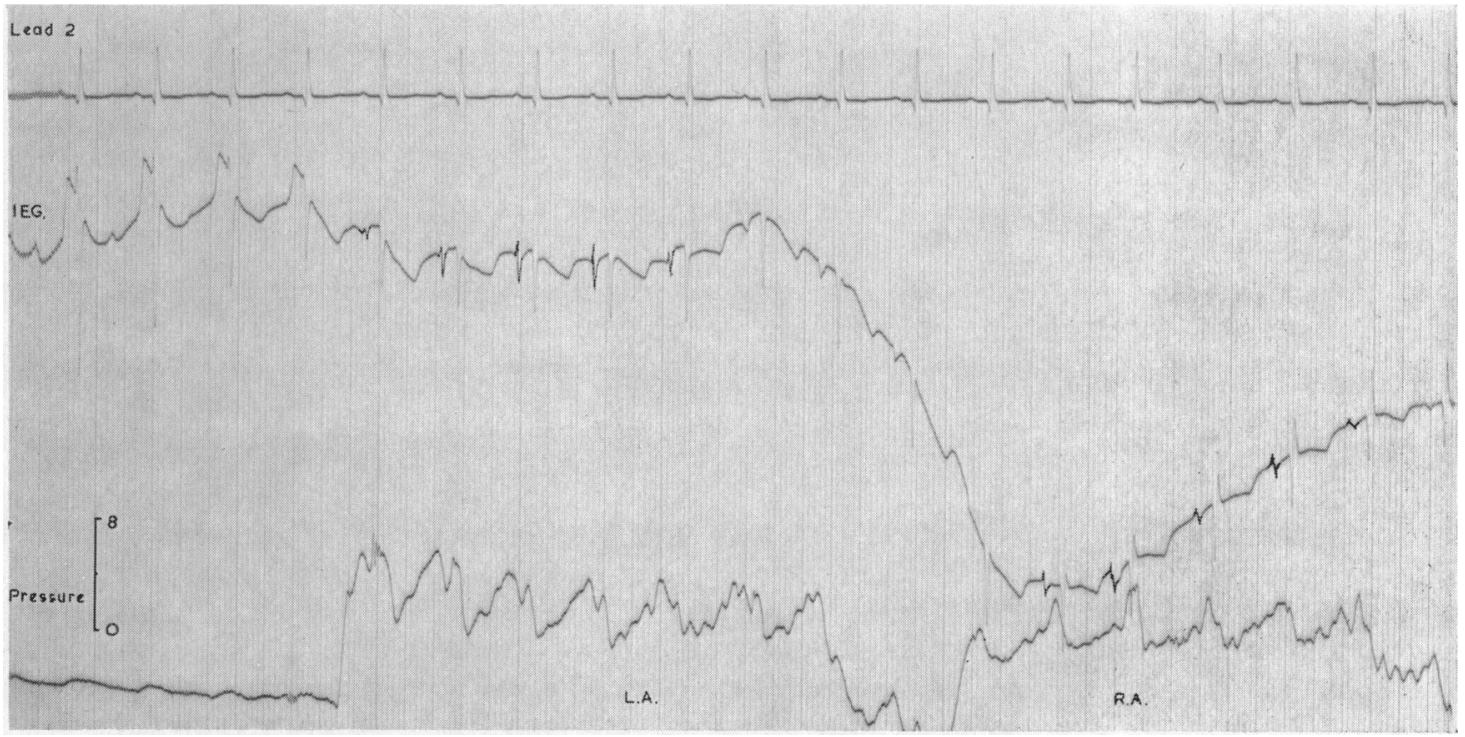

Fig. 4.-A withdrawal tracing from left to right atrium through an atrial septal defect, where initially the tip of the catheter has been pressed against the lateral wall of the left atrium producing atrial injury or contact currents and damping of the pressure pulse. This illustrates how, once the site of the defect has been pin-pointed on the record by the catheter flip, the polarity of the $\mathbf{P}$ wave can be studied on either side of the defect. In this case they are biphasic in the left atrium and biphasic in the right atrium, suggesting that the catheter has crossed in the mid septal zone through a defect of secundum type. 
say much about the nature of the deflections as the catheter actually crosses the septum and at this time too there may be a few atrial ectopic beats which obscure the true nature of the $P$ waves on the right atrial side of the defect (Fig. 5). If several slow and careful withdrawals are made, however, there is nearly always one which is free of such interference.

When a catheter passes out into a pulmonary vein it is often difficult to tell, even with careful screening and multiple blood sampling, whether it has done so from left or right atrium. The origin of the vein is usually well inside the edge of the heart shadow and the site of the transition from vein to atrium impossible to determine visually. If the catheter is not withdrawn far enough the blood still comes from the pulmonary vein and, the sample being mistakenly thought to be a left atrial one, an anomalous vein may be missed. If on the other hand it is withdrawn a little too far in cases where the atrial septum lies obliquely, the tip may be pulled unwittingly from left to right

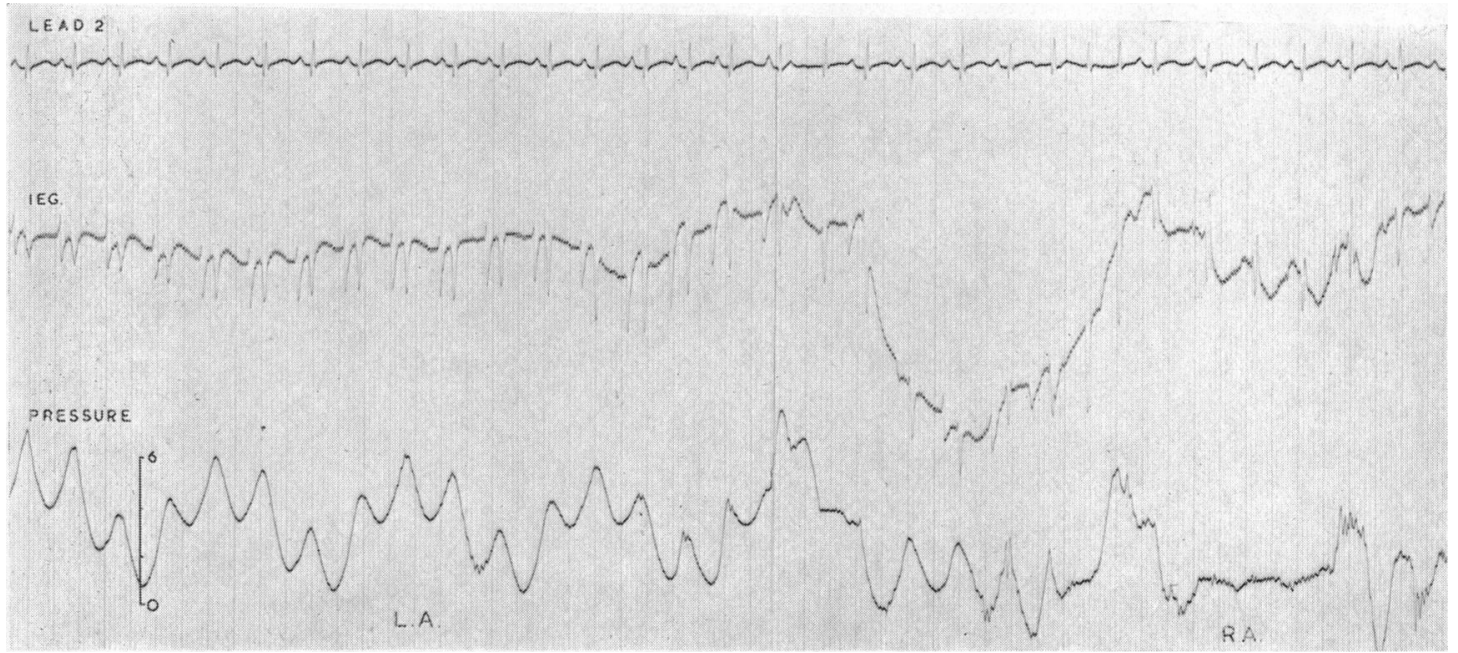

FIG. 5.-A withdrawal tracing from left to right atrium through foramen ovale, where initially the tip of the catheter is in mid left atrium. This shows the change from biphasic to negative $P$ waves as, on withdrawal, the catheter tip rises up in the atrium. The base-line movement indicates that it has slipped back through the septum into right atrium, the negative $P$ waves indicating that it has crossed high in the septal wall through the foramen ovale.

atrium and a normally sited vein may be thought anomalous. The nature of the complexes recorded from the electrode at the tip of the catheter can help on these occasions because the venogram and atriogram are often different and if the withdrawal is carefully monitored it is then easy to spot the first complex recorded inside the heart. A blood sample taken there, and the nature of this complex when the record is analysed, leave little doubt about the site of origin of the vessel. Fig. 6 illustrates the change which takes place in an intracardiac electrogram as a catheter is pushed out of the heart from left atrium into a pulmonary vein and then pulled back in again. The morphology of the complexes changes as the catheter passes from one to the other and at the same time there is movement of the baseline as the catheter tip flips over the edge of the atrial wall at the mouth of the vessel. It is important to realize that it is impossible to tell from which atrium an isolated complex has been recorded as neither the atrial nor the ventricular components of the atriogram are in 


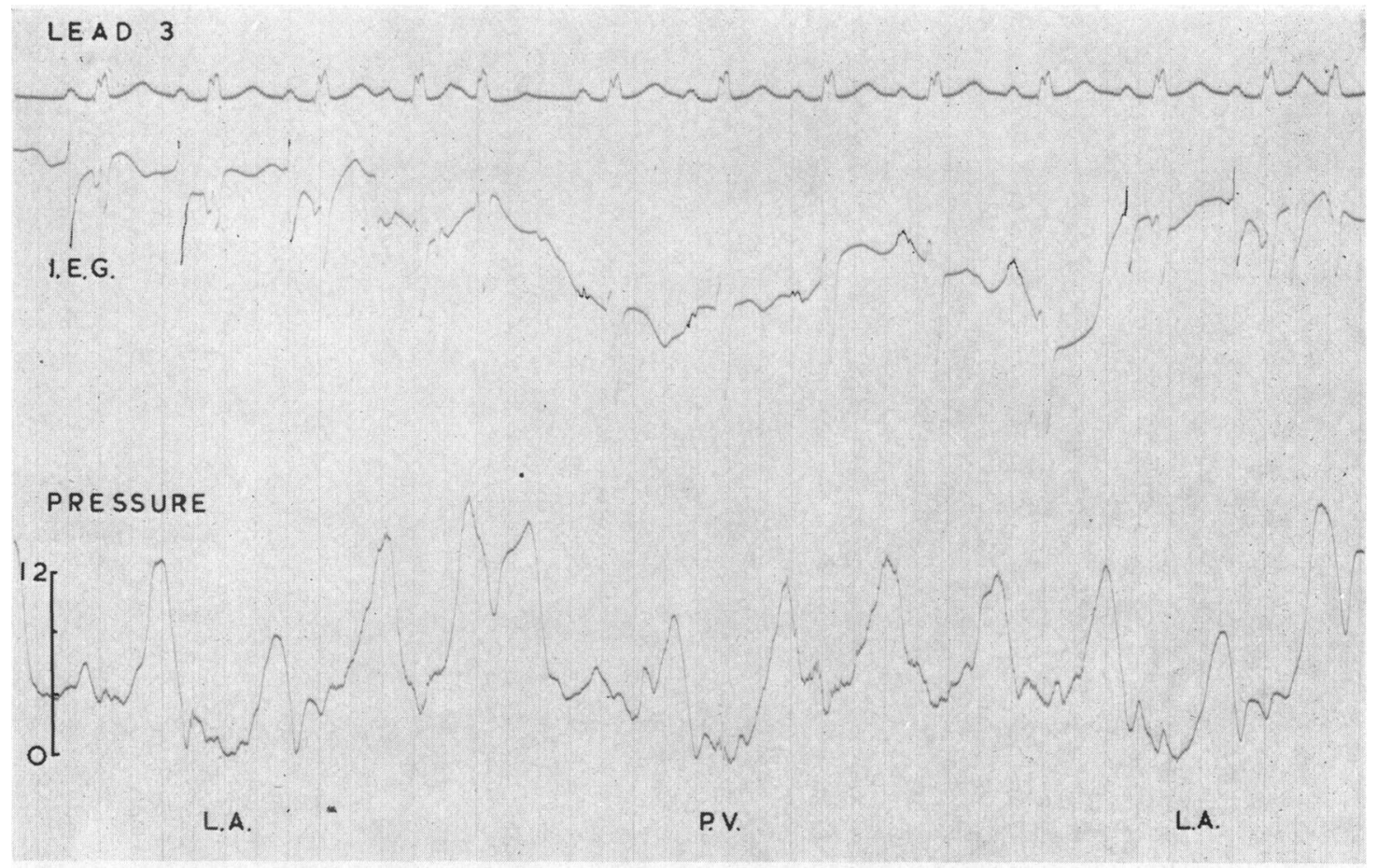

FIG. 6.-Illustrating how the complexes recorded by the electrode at the tip of the catheter change as it passes out of the left atrium into a pulmonary vein and is then pulled back in again. The first intracardiac complex can thus be used to position the catheter for blood sampling to determine the site of origin of the vessel.

any way specific for the right or left atrium. In this illustration for example, the intrinsic deflection of the $\mathrm{P}$ wave occurs early in the $\mathrm{P}$ wave component of the simultaneously recorded standard lead II, and though in the left atrium this deflection is often late, it is frequently no later than the initial deflection in the right atrium. Similarly though the ventricular component in the left atrium usually picks up a $Q$ from the cavity of the left ventricle, it is in no way unusual for it to have an initial positive deflection or for $Q$ waves to be recorded in the right atrium. It is only possible to be certain where a complex was recorded when it is studied in detail along with the other records made during the catheterization. 


\section{VENTRICULOGRAMS}

The morphology of the complexes usually recorded in the ventricles is illustrated in Fig. 7. In the right ventricle there is an $\mathrm{rS}$ pattern and though certain variations are not uncommon the main deflection in the right ventricular cavity is normally negative. The height of $r$, however, is variable and it may even be absent altogether in some cases-especially where the pressure is high. On the other hand, it is sometimes the main deflection in the outflow tract where a late $\mathrm{R}$ may be seen close to the pulmonary valve. Too little attention has been paid to the varying electrical forces in the right ventricular outflow tract perhaps because they are difficult to record. On most withdrawal tracings the catheter, once through the pulmonary valve, passes rapidly down into the right ventricular cavity so that at best there are only one or two complexes recorded under the valve

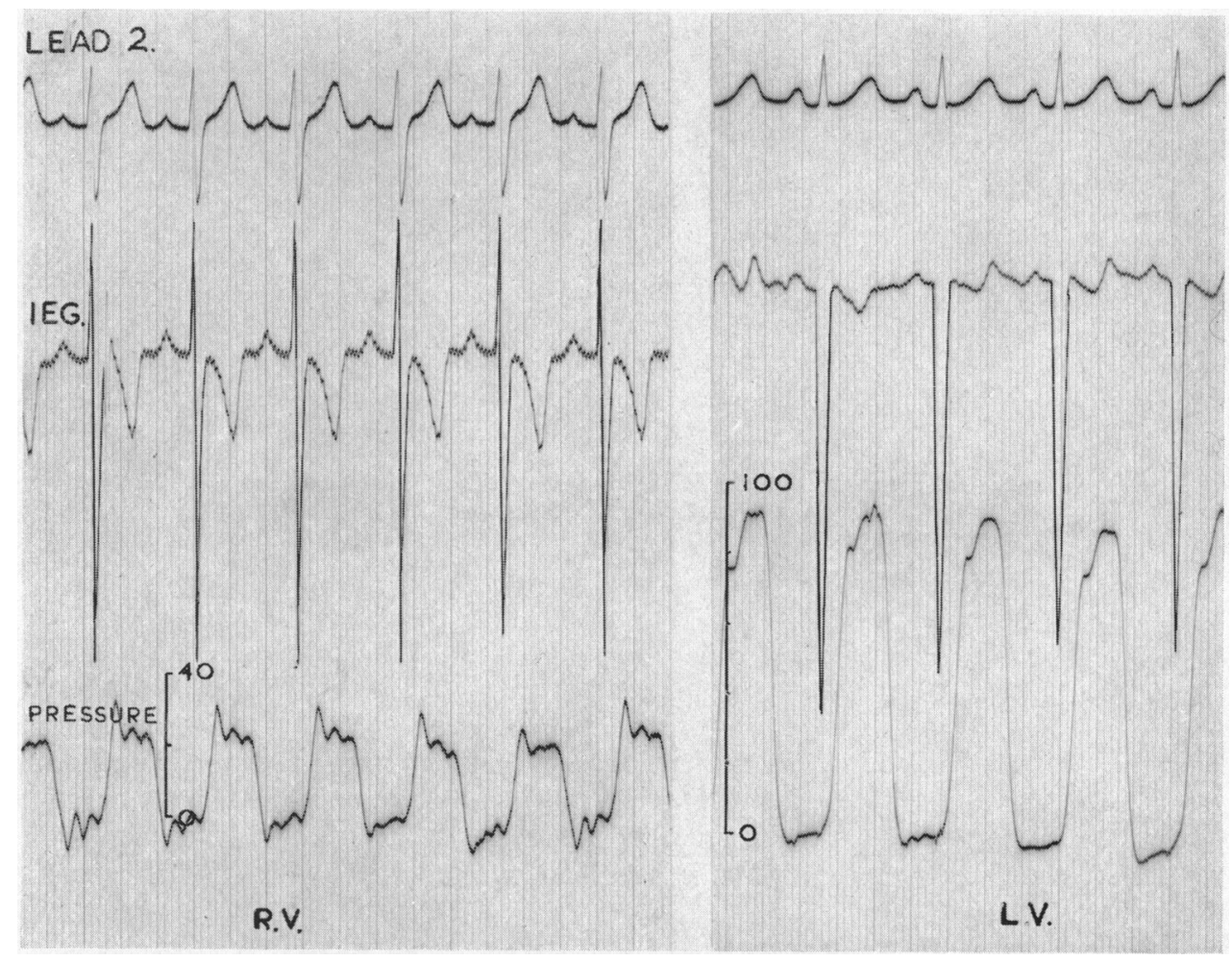

FIG. 7.-Showing the normal morphology of the intracardiac electrogram in the ventricles. In the right ventricle there is usually an $r$ wave of varying height and a deep $S$ wave while in the left ventricle a deep $Q$ is the sole deflection.

and the rest are cavity potentials. If the catheter is withdrawn slowly, the tip tends to scrape down against the wall during its descent through the outflow tract producing multiple ectopic beats or short bursts of ventricular tachycardia which obscure the normal morphology. A deep Q is as a rule the sole deflection in the left ventricle and though $\mathrm{r}$ waves have occasionally been seen in this chamber they are rare and unexplained.

Many babies requiring investigation already have a fully developed Eisenmenger reaction so that there is little difference between the systolic pressures in the right and left ventricles, or in the pulmonary artery for that matter if there is no pulmonary stenosis. Because these pressures are 
high, the heart rate rapid and the catheter size necessarily small, there is often considerable damping of the recorded pressure pulses, particularly of end-diastolic pressures which may be on the high side anyway because of a failing heart. For these reasons the interpretation of pressure pulse tracings can be difficult or even misleading and simultaneously recorded intracardiac electrograms can be most helpful. Fig. 8 is a record made as a catheter tip is withdrawn from left to right ventricle through a ventricular septal defect. Though the damped pressure-pulse tracing gives no indication of this, the intracardiac lead shows clearly what has happened-there are deep Q waves in the left ventricular cavity, a couple of extrasystoles as the catheter tip scrapes through the defect and the appearance of initial $r$ waves immediately the right ventricle is entered.

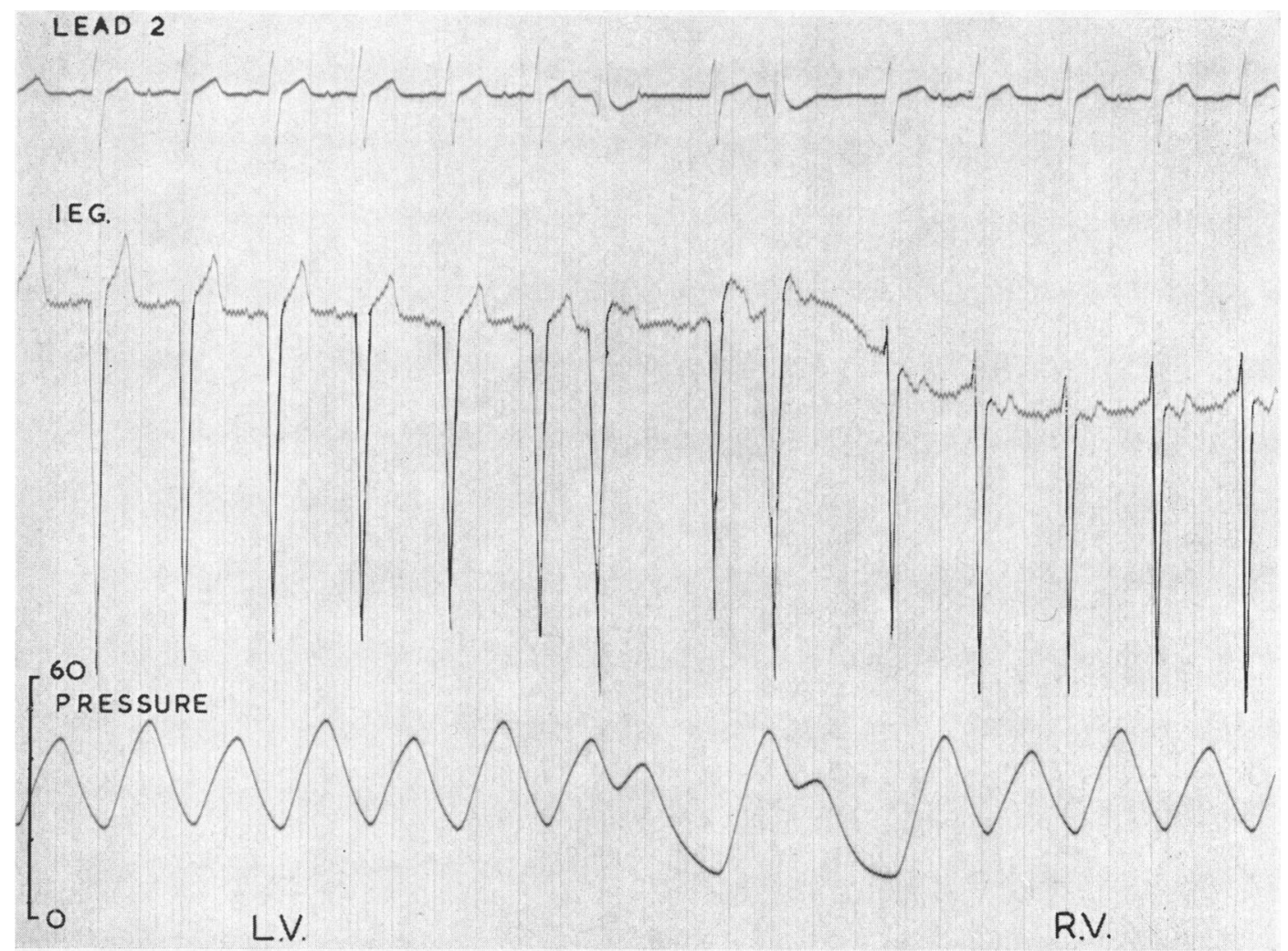

Fig. 8.-A withdrawal tracing from left to right ventricle through a ventricular septal defect in an infant with the Eisenmenger syndrome. This shows how in the absence of any obvious change in the pressure pulse the complexes recorded from the intracardiac lead indicate the course taken by the catheter. There are deep $Q$ waves in the left ventricle, extrasystoles as the tip scrapes through the defect and $r$ waves appear as soon as the cavity of the right ventricle is entered.

It is often impossible to catheterize the right or left pulmonary arteries from the groin in small children - the catheter lies pointing upwards in the upper part of the cardiac silhouette and it is difficult to be sure on the X-ray screen whether the tip has actually passed through the pulmonary valve or merely lies in the upper part of the right ventricular outflow tract. When a withdrawal is made the pressure pulse may suggest severe pulmonary stenosis (Fig. 9) but study of the intracardiac. electrogram may demonstrate conclusively that no such lesion is present. The complexes are all typically right ventricular and it is clear from the S-T shifts or ventricular contact currents in the first part of the record, that the catheter tip has been pushed up under the pulmonary valve 
cusps causing pressure damping. This disappears when contact is lost and the pressure tracing thus simulates that of pulmonary stenosis.

Pressure damping, also, may obscure the diastolic gradient between the pulmonary artery and the right ventricle and Fig. 10 illustrates a case in which, without the aid of the intracardiac lead, it would be difficult to be certain that the catheter had in fact passed from pulmonary artery to right ventricle. The transition from the low voltage complexes recorded in the thin walled great vessel to the high voltage and obviously right ventricular complexes in the thick muscular walled chamber dispels any doubts and confirms the absence of pulmonary stenosis in such cases.

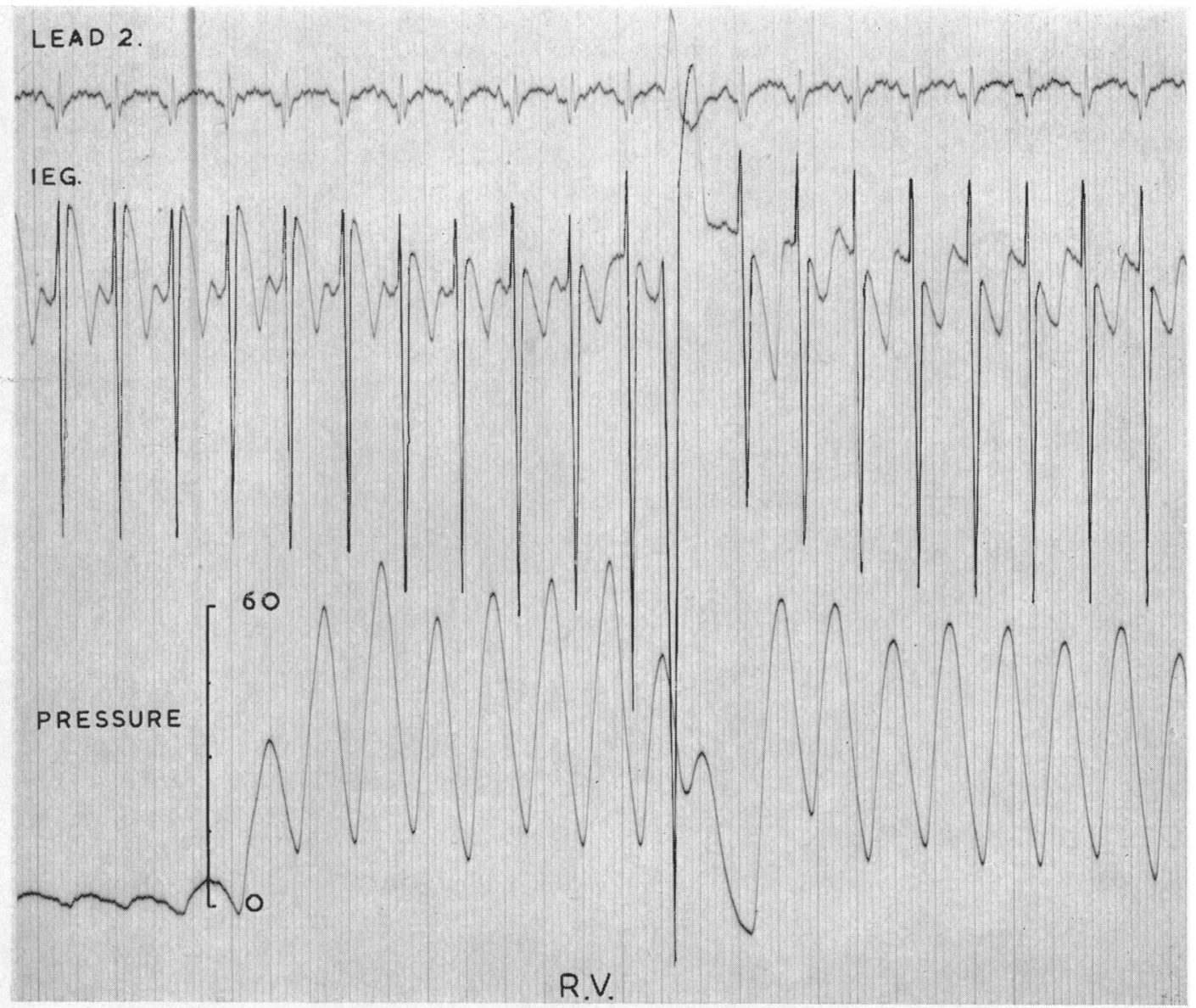

FIG. 9.-A record made as a catheter was withdrawn from what was thought to be pulmonary trunk into the right ventricle and where the pressure pulses suggest pulmonary stenosis. The intracardiac electrogram shows that the catheter has never been out of the right ventricle and the S-T elevations indicate that the tip has been pushed up under the pulmonary valve cusps with consequent damping of the pressure pulse. As withdrawal begins contact is lost, the $\mathbf{S}-\mathbf{T}$ elevations disappear and the right ventricular pressure pulse reappears. 


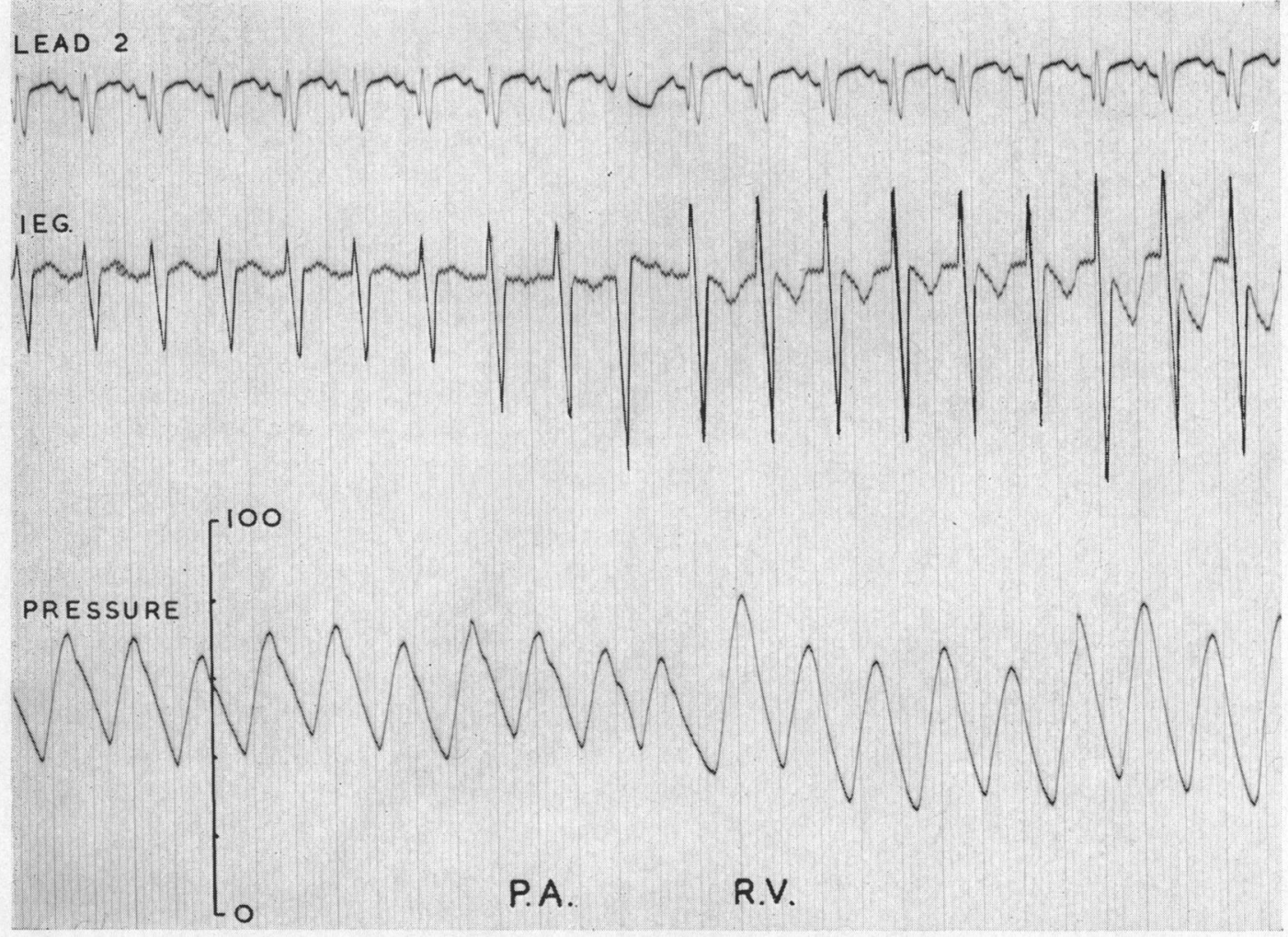

FIG. 10.-Illustrating how damping may obscure the diastolic gradient between the pulmonary arterial and right ventricular pressure pulses in cases with the Eisenmenger reaction. The changing complexes recorded by the intracardiac electrode, however, confirm the route taken by the catheter in such cases.

\section{ANGIOCARDIOGRAPHY}

In these days, when contrast medium is injected directly into the heart under high pressure, it is very important to be quite certain where the tip of the catheter is before the injection is made. If it is not precisely in the selected site the desired films will not be obtained and serious damage may be caused to the heart. It is usual to position the catheter by X-ray screening and to confirm its site by examination of the pressure pulse, but in the tense moments before an angiocardiogram is fired the wave form may easily be misinterpreted and a pressure pulse of the type seen in Fig. 11 may easily be mistaken for a ventricular one. On careful analysis it is obviously a giant "a" wave, and had the intracardiac electrogram also been on the oscilloscope it would have been clear that the catheter was in the atrium where the complexes are quite different from those usually seen in the ventricle (Fig. 12).

Perhaps the greatest danger is from injections of contrast medium under high pressure into the coronary sinus, where the pressure pulse may closely resemble a right ventricular one (Fig. 13). 


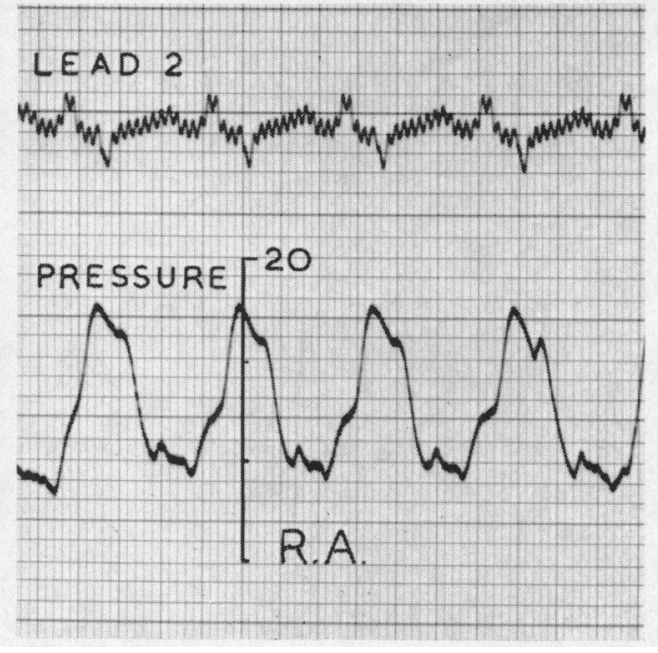

FIG. 11.-A giant "a" wave which was mistaken for a right ventricular pressure pulse before injection of opaque medium at angiocardiography.
The electrogram recorded in the coronary sinus is however so characteristic that such errors should be easily avoided if it is used routinely as well as the pressure pulse to check the site of the catheter tip before injections are made.

We have had one or two rather alarming experiences, fortunately none of them fatal, where, because of the high pressure required to force contrast through small lumen catheters, some of the contrast medium has been forced under the endocardium. It is now our practice to check the position of the catheter tip in the right ventricle very carefully before injections are made by examining the complexes from the intracardiac lead for $\mathrm{S}-\mathrm{T}$ elevations (Fig. 14A). If these are present it means that the catheter tip is either pressed against the ventricular wall or buried in the columnæ carneæ. The catheter is withdrawn until they disappear and normal ventricular complexes return to the oscilloscope (Fig. 14B)-signifying that contact has been lost and that the tip of the catheter now lies free in the ventricular cavity where injection may be more safely made.

\section{Discussion}

The investigation of small children is often a matter of some urgency and Keith (1956) has shown that by far the highest mortality among children with congenital heart disease is in the first year of life. Surgical treatment has in recent years improved the prognosis in some conditions which may be lethal at this age, and infants with coarctation of the aorta and patency of the ductus arteriosus are now operated upon routinely when necessary. The more complex deformities still present formidable problems to the surgeon, even in older children, but the repair of simple lesions, such as ventricular septal defect, awaits only the perfection of a suitable method of perfusion. Ventricular septal defects are often badly tolerated in infancy. Some babies with this lesion rapidly develop heart failure and die; others while failing to thrive can be tided along with careful medical treatment but succumb to intercurrent infections; a number, however, after a bad start, seem to adapt themselves quite well to their Eisenmenger reaction and will grow big enough for an operation which may be hazardous or even contra-indicated because of their altered pulmonary hæmodynamics.

There seems little doubt that before long this surgical challenge will be mastered and as exact diagnosis is not always easy in very small children our investigative methods require to be developed not only to select those for whom surgical treatment is already possible but also those for whom it soon will be. In infants cardiac catheterization may have to be abandoned at any stage, or a catheter which has taken an unusual route may have to be somewhat hastily withdrawn before pressure records or oxygen saturations can be recorded. It is wise to record every bit of information as soon as it becomes available because, for a variety of reasons, once a catheter has been withdrawn it may not be possible to get it back again. From the preceding illustrations it will be apparent that the routine use of electrode catheters can in many ways be of the greatest possible assistance both during and after such procedures.

When time is limited it may be decided that selective injection of contrast medium and cineangiocardiography (Watson et al., 1958) will be of greater value than continued catheterization, and 


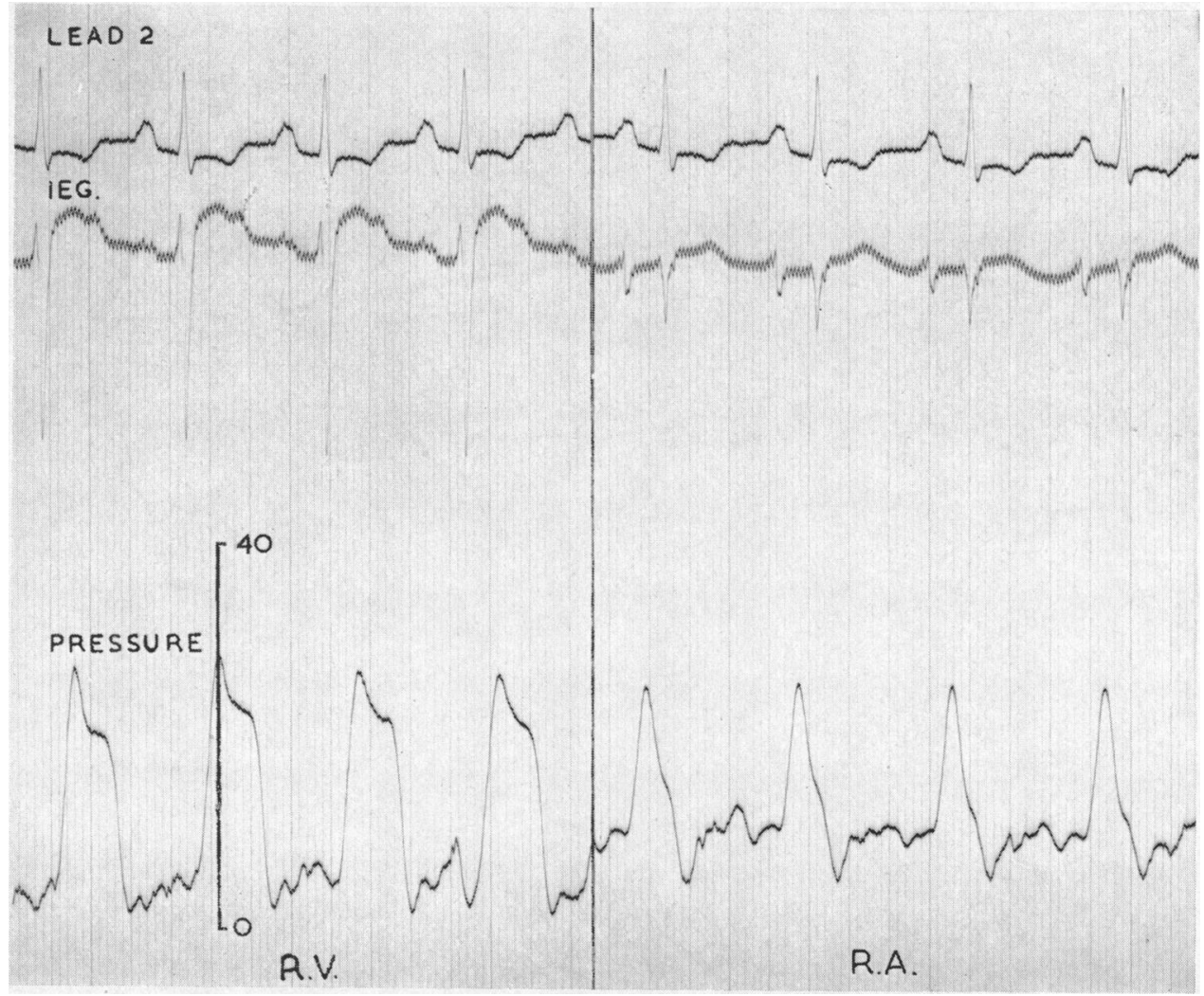

FIG. 12.-From a case of tricuspid stenosis. The record shows that even where the right ventricular and right atrial pressure pulses are of similar magnitude, it is easy to tell from which chamber a pressure pulse comes when there is a simultaneously recorded intracardiac electrogram.

\section{LEAD 2.}

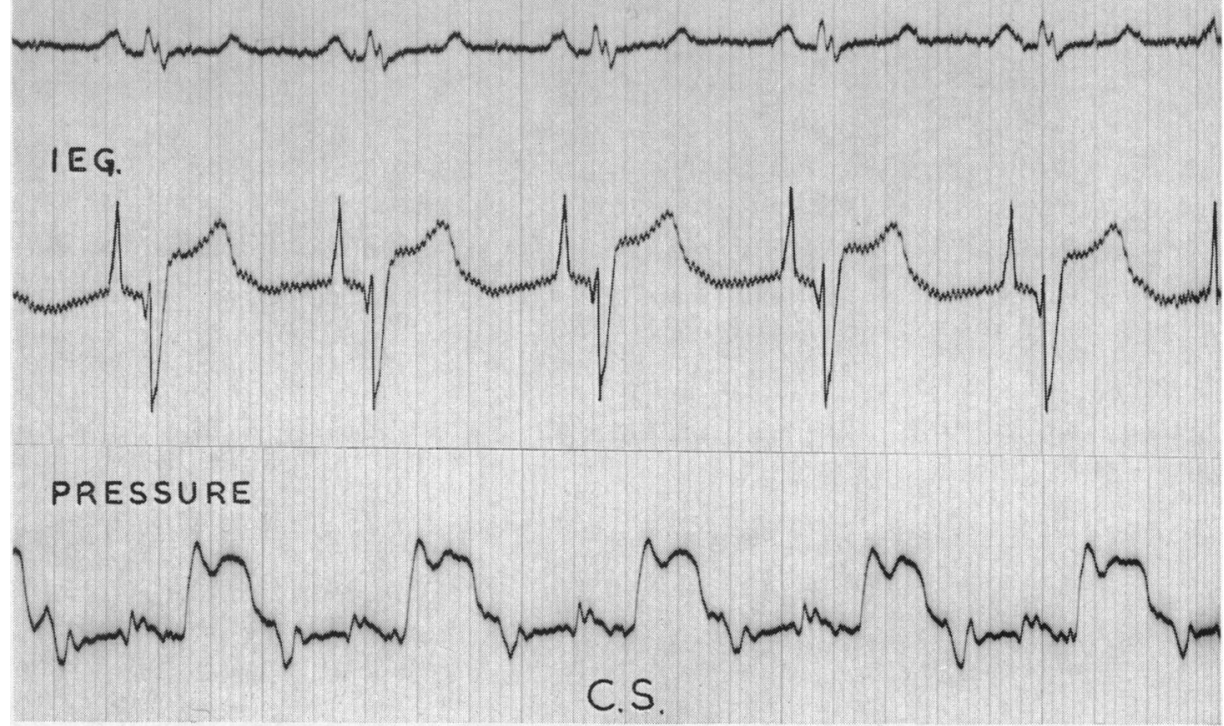

FIG. 13.-Showing that though the pulse wave in the coronary sinus is often indistinguishable from a right ventricular one-the intracardiac electrogram is quite characteristic and its appearance should prevent accidental injection of contrast media at this site. 


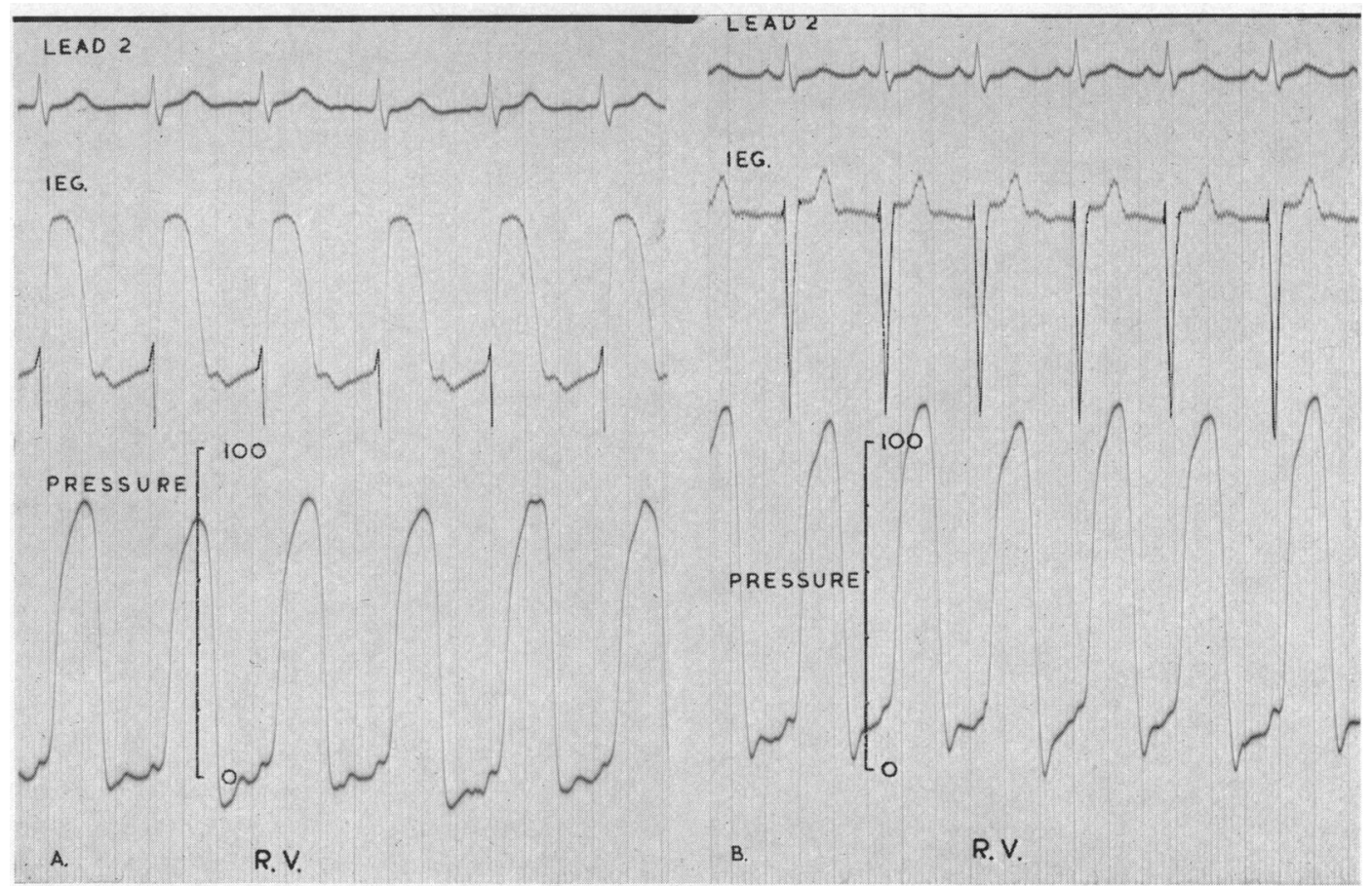

FIG. 14.-(A) Illustrating the gross S-T elevations which appear on the intracardiac electrogram when the tip of the catheter is pressed against the ventricular wall during siting before angiocardiography. (B) The catheter has been withdrawn, contact has been lost and the return of the normal right ventriculogram indicates that the tip now lies free in the ventricular cavity where injection may be more safely made.

here too the intracardiac lead will help with the correct positioning of the catheter so that the maximum information will be obtained in the safest possible way.

\section{SUMMARY}

The high mortality during the first year of life amongst children with congenital heart disease demands that more attention be paid to those in this age group. Diagnostic methods require some modification for their investigation and the electrogram recorded inside the heart by an electrode at the tip of the catheter is most helpful. This paper illustrates a few of the ways in which it may make cardiac catheterization in infants and the newborn quicker, safer and more informative.

I would like to record my thanks to Professor Ian G. W. Hill for his continuing help and encouragement, and to my many pædiatric colleagues for asking me to help with the investigation and treatment of these little children.

\section{REFERENCES}

Emslie-Smith, D. (1955). Brit. Heart J., 17, 219.

Keith, J. D. (1956). Padiatrics, 18, 491.

Watson, H., Pickard, C., Lowe, K. G., and Hill, I. G. W. (1958). Brit. Heart J., 20, 459. 